\section{RNA-guided gene editing}

Targeted gene modification can be guided by programmable RNA in bacteria, zebrafish and mammalian cells.

Humble creatures, prokaryotes and viruses, have an illustrious history of providing biologists with molecular tools. Where, after all, would molecular biology-or for that matter, all of genomicsbe without restriction endonucleases or DNA polymerases? And, as a recent flurry of papers reporting RNA-guided genome engineering demonstrates, the bacteria are still at it.

The clustered, regularly interspaced, short palindromic repeats (CRISPR) system is a component of bacterial and archaeal immunity. The CRISPRassociated (Cas) endonuclease cleaves foreign nucleic acids, directed to its target sequence by two small RNAs. In work published last year, researchers showed that the CRISPR-Cas system of Streptococcus pyogenes could be programmed to direct in vitro cleavage of desired target sequences. This raised the possibility that the approach could be used for targeted gene editing in vivo. With an almost startling rapidity, five recently published papers now show that it can.

The groups of George Church at Harvard Medical School, Feng Zhang at the Broad Institute and Jin-Soo Kim at Seoul National University describe methodological variations on essentially the same theme: the $S$. pyogenes Cas9 nuclease, together with two noncoding RNAs (the CRISPR RNA (crRNA) and trans-activating crRNA (tracrRNA)), effects the cleavage of endogenous sequences in mammalian cells.

The two RNAs can be provided either in chimeric form or as two separate molecules; they may be transcribed from a plasmid or transcribed in vitro prior to delivery into the cell. Importantly, in all cases, the target sequence is specified by complementarity to the 20-base-pair crRNA. The minimal constraint on the sequence is the requirement for the so-called protospacer adjacent motif (PAM), which takes the form NGG, immediately following the 20-base-pair target sequence.

In contrast to current tools, zinc-finger nucleases and transcription activator-like effector nucleases (TALENs), targeting of a new sequence with CRISPR-Cas requires the design of only a new RNA guide and not a new pair of enzymes. The efficiency of cleavage of mammalian genes reported in these studies is equivalent to or better than that seen with TALENs. The Church and Zhang groups report mutations induced by nonhomologous end joining-based repair at the cleavage site as well as sequence introduction by homologous recombination from a donor construct (Mali et al., 2013; Cong et al., 2013). The Kim group derived clonal mutant cell lines by limiting dilution and without antibiotic selection, which suggests that the system can be well tolerated by cells (Cho et al., 2013).

The group of Keith Joung and colleagues, also at Harvard Medical School, have applied RNA-guided editing to mutate endogenous genes in the zebrafish (Hwang et al., 2013), achieving mutation at $>80 \%$ of targeted sites. Although they report no major toxic effects on embryos, phenotypes in adult fish and transmission of the mutations to the next generation remain to be examined.

The fact that clonal cell lines can be derived and that there is no unexpected toxicity in the fish embryo suggests that RNA-guided nucleases (RGENs) do not cleave indiscriminately in eukaryotic cells. But what about their finer specificity? How likely are RGENs to cut at related off-target sites? A fifth study from Luciano Marraffini and colleagues at The Rockefeller University offers some sobering observations.

Marraffini and colleagues use the CRISPR system for RNA-guided editing of bacterial genomes, applying this both to the recombinogenic Streptococcus pneumoniae and, in tandem with recombineer-

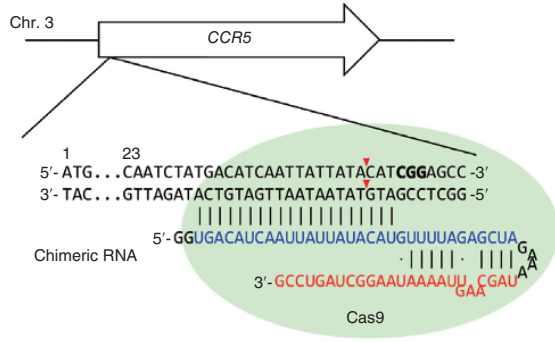

RNA-guided editing. The schematic depicts Cas9 (green) cleavage of a target site in the CCR5 gene, mediated by a chimeric guide RNA. Image adapted from Nature Biotechnology.

ing, to Escherichia coli (Jiang et al., 2013). As part of this work, the researchers studied the sequence requirements for target cleavage.

They found that single nucleotide changes at every position in the 12 PAMproximal nucleotides of the target sequence can abrogate cleavage, but not every change does so to the same degree. And though the PAM sequence is critical for cleavage, some single-nucleotide changes are tolerated (albeit with reduced cleavage). Although these experiments were conducted in bacteria and results from the other groups in the eukaryotic context and in vitro suggest that RGENs are specific, this is an area that will benefit from more examination.

What remains eminently clear is that prokaryote biology is still fertile hunting ground for new laboratory methods.

\section{Natalie de Souza}

\section{RESEARCH PAPERS}

Cho, S.W. et al. Targeted genome engineering in human cells with the Cas9 RNA-guided endonuclease. Nat. Biotechnol. advance online publication (29 January 2013). Cong, L. et al. Multiplex genome engineering using CRISPR/Cas systems. Science published online (3 January 2013).

Hwang, W.Y. et al. Efficient genome editing in zebrafish using a CRISPR-Cas system. Nat. Biotechnol. advance online publication (29 January 2013). Jiang, W. et al. RNA-guided editing of bacterial genomes using CRISPR-Cas systems. Nat. Biotechnol. advance online publication (29 January 2013).

Mali, P. et al. RNA-guided human genome engineering via Cas9. Science published online (3 January 2013). 